OPEN ACCESS

Edited by:

Zongxin Ling,

Zhejiang University, China

Reviewed by:

Zhenlin Han,

University of Hawaii at Manoa,

United States

Zhao Chen,

University of Maryland, College Park,

United States

Fengli Guo,

Stowers Institute for Medical

Research, United States

*Correspondence:

Wei Chen

chenwei66@jiangnan.edu.cn

Specialty section:

This article was submitted to Microbiome in Health and Disease,

a section of the journal

Frontiers in Cellular

and Infection Microbiology

Received: 18 January 2021

Accepted: 23 February 2021

Published: 18 March 2021

Citation:

Qiao Y, Qiu Z, Tian F, Yu L, Zhao J, Zhang H, Zhai $Q$ and Chen W (2021)

Pediococcus acidilactici Strains

Improve Constipation Symptoms and

Regulate Intestinal Flora in Mice.

Front. Cell. Infect. Microbiol. 11:655258.

doi: 10.3389/fcimb.2021.655258

\section{Pediococcus acidilactici Strains Improve Constipation Symptoms and Regulate Intestinal Flora in Mice}

\author{
Yiteng Qiao, ${ }^{1,2}$,Zhichang Qiu $^{3}$, Fengwei Tian ${ }^{1,2}$, Leilei Yu ${ }^{1,2}$, Jianxin Zhao ${ }^{1,2}$, \\ Hao Zhang ${ }^{1,2,4,5}$, Qixiao Zhai ${ }^{1,2}$ and Wei Chen ${ }^{1,2,4 *}$ \\ ${ }^{1}$ State Key Laboratory of Food Science and Technology, Jiangnan University, Wuxi, China, ${ }^{2}$ School of Food Science and \\ Technology, Jiangnan University, Wuxi, China, ${ }^{3}$ College of Food Science and Engineering, Shandong Agricultural University, \\ Tai'an, China, ${ }^{4}$ National Engineering Research Center for Functional Food, Jiangnan University, Wuxi, China, ${ }^{5}$ Wuxi \\ Translational Medicine Research Center, Jiangsu Translational Medicine, Research Institute Wuxi Branch, Wuxi, China
}

Constipation is a prevalent gastrointestinal disorder that seriously reduces the quality of life. Clinical studies have shown that a great change or severe imbalance occurs in the intestinal microbiota of people with constipation. This study explored whether bacteriocinproducing and non-bacteriocin-producing Pediococcus acidilactici strains resulted in differences in the alleviation of constipation and changes in the fecal flora in BALB/c mice. The constipation-related indicators, gastrointestinal regulatory peptides and gut microbiota were identified to evaluate their alleviating effects and underlying mechanisms. The time to the first black-stool defecation and the gastrointestinal transit rate in constipated mice were found to be somewhat improved by four $P$. acidilactici strains $(P>0.05)$. Moreover, there were significant differences in the level of most gastrointestinal regulatory peptides in the serum, as well as in the composition and abundance of intestinal microbiota in different groups $(P<0.05)$. At the phylum level, the relative abundance of Firmicutes was significantly increased, but those of Bacteroidetes and Proteobacteria were significantly reduced after the administration of four $P$. acidilactici strains for $14 \mathrm{~d}(P<0.05)$. The levels of Bacteroides and genera from Enterobacteriaceae were significantly decreased, whereas Bifidobacterium and Lactobacillus were upregulated when bacteriocin-producing $P$. acidilactici CCFM18 and CCFM28 strains were provided in the diet $(P<0.05)$. The results indicated that although constipationrelated symptoms were alleviated to only a limited degree, the administration of four $P$. acidilactici strains effectively regulated the gut flora and provided a potential health benefit to the host, especially the bacteriocin-producing $P$. acidilactici strains. 


\section{INTRODUCTION}

Constipation, a common gastrointestinal disorder, is characterized by low defecation frequency, prolonged emptying time of the gastrointestinal tract, and incomplete defecation, which causes serious distress for patients of different ages and genders (Bharucha, 2007; Zhao and Yu, 2016). Globally, the prevalence of constipation is showing an increasing trend with changes in dietary habits, lifestyle, and psychological factors. The treatment of constipation is challenging. Irritant drugs have limited efficacy and serious adverse effects (Bharucha et al., 2017; Luthra et al., 2019). Therefore, oral supplementation with probiotics (safe and long-acting) is attracting considerable interest among gastrointestinal physicians and researchers (Dimidi et al., 2020).

The term probiotics refers to live microorganisms that are introduced to the gastrointestinal tract of the host with beneficial effects (Bezkorovainy, 2001). The oral intake of probiotics cultured in vitro prompts regulation of the composition and abundance of intestinal flora and the metabolism of short-chain fatty acids (SCFAs) by healthy colonic populations to inhibit the growth of pathogens, reduce the concentration of several metabolic products (such as some phenols), lower the intestinal $\mathrm{pH}$, and promote intestinal peristalsis (Possemiers et al., 2009). In a double-blind placebo-controlled, randomized study of 45 children with chronic constipation, the administration of Lactobacillus casei rhamnosus Lcr35 resulted in increased defecation frequency, enhanced treatment success rates, decreased use of glycerin enemas, and reduced number of hard stools, compared with the placebo group (Bu et al., 2007). The beneficial effect of the consumption of probiotics has also been examined in adults and the elderly (Zaharoni et al., 2011; Eskesen et al., 2015).

Pediococcus acidilactici probiotic strains have been proven to produce bacteriocin from ribosomes. Since the 1980s, researchers have conducted many studies on the discovery and production of bacteriocin. Bacteriocins can colonize and persist in the human gut while having a beneficial effect on the host, including modulation of the composition of the gut microbiota, improvement of the host immune response, and enhancement of the gut barrier function (Corr et al., 2007; Walsh et al., 2008; Kommineni et al., 2015; Umu et al., 2016; Heeney et al., 2019). Gonzalez and Kunka (1987) reported that a 6.2-megadalton plasmid was responsible for the production of bacteriocin in $P$. acidilactici PAC1.0, designated as pediocin PA-1, which had a molecular weight of ca. 16,500. Subsequently, a variety of bacteriocins have been discovered and identified, including pediocin Ach, pediocin JD1, pediocin SJ1, and pediocin N5P. At present, $P$. acidilactici strains are widely used in the food industry, animal husbandry, and medical settings. The bacteriocins produced are known to inhibit the growth of pathogenic microorganisms in the host or to act as signalregulated peptides to regulate the health of the host (Chelliah et al., 2020). Although many studies have confirmed the promising effects of several probiotics in the treatment of constipation, the degree of alleviation provided by bacteriocinproducing $P$. acidilactici strains and their regulatory roles on the intestinal flora remain poorly understood. Therefore, it is necessary to investigate the effects of bacteriocin-producing and non-bacteriocin-producing $P$. acidilactici strains on constipation-related symptoms and the composition of the intestinal flora.

In this study, loperamide hydrochloride was used to induce constipation in mice, which were then treated with different $P$. acidilactici strains. The constipation-related symptoms were identified to evaluate the effects of different strains on the constipation. Besides, to explore the underlying mechanism of the effect of the P. acidilactici strains on constipation, an enzymelinked immunosorbent assay and a high-throughput MiSeq sequencing technique were used to analyze the levels of gastrointestinal regulatory peptides and the composition of the fecal microbiota, respectively.

\section{MATERIALS AND METHODS}

\section{Chemicals and Reagents}

The levels of motilin (MTL), gastrin (Gas), substance P (SP), endothelin (ET), somatostatin (SS) and vasoactive intestinal peptide (VIP) were measured using a kit purchased from Wen LE Bioengineering Institute (Shanghai, China). MRS broth was obtained from Qingdao Hope Bio-Technology Co., Ltd (Qingdao, China). Loperamide hydrochloride (2 mg per capsule) was obtained from Wuxi Pharmacy (Xi'an Janssen Pharmaceutical Ltd., Xi'an, China). Loperamide hydrochloride was dissolved in distilled water to a final concentration of $1 \mathrm{mg} /$ $\mathrm{mL}$. Phenolphthalein ( $0.1 \mathrm{~g}, 100$ tablets) was dissolved in distilled water to a final concentration of $7 \mathrm{mg} / \mathrm{mL}$.

For the activated carbon meal solution, we added $100 \mathrm{~g}$ of gum arabic to $800 \mathrm{~mL}$ of water, which was boiled until the solution became transparent. We then added $50 \mathrm{~g}$ of activated carbon to the solution and boiled it three times. After the solution had cooled to room temperature, we added water to adjust the volume to $1000 \mathrm{~mL}$, stored it at $4^{\circ} \mathrm{C}$, and mixed it prior to use. All other chemicals and reagents used in this study were of analytical grade.

\section{Bacterial Strains and Growth Media}

The bacteriocin-producing P. acidilactici CCFM28 strain was provided by the University of Groningen in the Netherlands, and $P$. acidilactici CCFM 18 strain was isolated from Chinese pickles. The non-bacteriocin-producing $P$. acidilactici NT17-3 and $102 \mathrm{H} 8$ strains were isolated from human feces.

To activate and amplify the $P$. acidilactici strains, an MRS medium was used that contained $10.0 \mathrm{~g}$ of peptone, $5.0 \mathrm{~g}$ of beef extract, $4.0 \mathrm{~g}$ of yeast extract, $20.0 \mathrm{~g}$ of glucose, $2.0 \mathrm{~g}$ of $\mathrm{K}_{2} \mathrm{HPO}_{4}$, $2.0 \mathrm{~g}$ of ammonium citrate, $5.0 \mathrm{~g}$ of sodium acetate, $0.2 \mathrm{~g}$ of $\mathrm{MgSO}_{4}, 0.05 \mathrm{~g}$ of $\mathrm{MnSO}_{4}$, and $1.0 \mathrm{~mL}$ of Tween 80 per liter. After adjusting the $\mathrm{pH}$ to $6.2 \pm 0.2$, we sterilized the medium at $115^{\circ} \mathrm{C}$ for $20 \mathrm{~min}$ and added $15.0 \mathrm{~g} / \mathrm{L}$ of agar to obtain a solid medium.

Four strains (including two bacteriocin-producing and two non-bacteriocin-producing strains) were used for intragastric administration to the constipated mice. Briefly, the 
$P$. acidilactici strains were activated at $37^{\circ} \mathrm{C}$ for $48 \mathrm{~h}$, and the bacterial suspensions were then centrifuged at 5,000 g for $15 \mathrm{~min}$ to generate deposits. After washing with phosphate-buffered saline three times, the clean bacterial deposits were dissolved in sterile skim milk solution, followed by lyophilization into a powder. The freeze-dried samples were then resuspended in physiological saline and adjusted to $10^{9} \mathrm{CFU} / \mathrm{mL}$ for administration to the mice.

\section{Animals and Sample Collection}

Seven-week-old female BALB/c mice, provided by the Shanghai Laboratory Animal Center (Shanghai, China), were kept in a standard rodent cage at a constant temperature and humidity under a strict 12-h light cycle. The mice were fed a commercial mouse diet and drinking water was freely available.

After being allowed to adapt to their new environment for one week, the mice were randomly divided into seven groups $(n=10$ for each group) (Battish et al., 2000). Apart from the normal control group, loperamide hydrochloride $(10 \mathrm{mg} / \mathrm{kg}, 200 \mu \mathrm{L})$ was intragastrically administered daily to the mice in the other six groups for $14 \mathrm{~d}$. Both the constipated and normal mice were fed a commercial diet and freely available drinking water. The mice in the $P$. acidilactici-treated group were given $200 \mu \mathrm{L}$ of bacterial suspension via intragastric infusion each day, including the bacteriocin-producing $P$. acidilactici CCFM28 and CCFM18 strains, and non-bacteriocin-producing $P$. acidilactici NT17-3 and $102 \mathrm{H} 8$ strains. In contrast, $200 \mu \mathrm{L}$ of physiological saline and phenolphthalein $(70 \mathrm{mg} / \mathrm{kg}$ body weight $)$ were given to the negative and positive control groups, respectively. The control groups were fed the control diet (FOS, 0\%) throughout the experiment (Lin et al., 2005). The body weights of the mice in all groups were recorded each day and their feces were collected daily for examination. After $14 \mathrm{~d}$ of treatment, the mice in all groups were fasted for $12 \mathrm{~h}$, although water was still available. Eyeball blood was then extracted to measure the level of gastrointestinal regulation peptides. After sacrificing the mice via cervical dislocation, the entire intestines were removed to measure the rate of advancement of ink through the small intestine. The contents of the colon and cecum were also collected and taken to the laboratory within $2 \mathrm{~h}$ for extraction of the intestinal genome.

\section{Detection of Constipation-Related Indicators}

To analyze the effects of different $P$. acidilactici strains on constipation in mice, we compared the time to the first blackstool defecation, using the method reported by Lee et al. (2012). Briefly, a mixture of ink and the corresponding test substances were given to the mice that had been constipated by loperamide hydrochloride, and the time to the first black-stool defecation for each mouse was recorded until the first black stools of all mice in the negative control group had been excreted.

The gastrointestinal transit rate provides information about intestinal motility and constipation-related symptoms. After the constipated mice had been intragastrically infused with a mixture of ink and the corresponding test substances for $30 \mathrm{~min}$, they were sacrificed via cervical dislocation. The gastrointestinal transit rate was calculated as follows (Nagakura et al., 1996):

$$
\begin{aligned}
& \text { Gastrointestinal transit rate }(\%) \\
& =\frac{\text { Ink advancement length }}{\text { The total length of the small intestine }} \times 100 \%
\end{aligned}
$$

\section{Analysis of Intestinal Microflora}

The FastDNA Spin Kit for Soil (MP Biomedicals, California, U.S.A.) was used to extract the microbial genomic DNA from the collected fecal samples. After quantifying the obtained DNA, a polymerase chain reaction (PCR) analysis of the V4 variable region of the bacterial $16 \mathrm{~S}$ rRNA was performed using the primers (forward primer, 5'-CCTAYGGGRBGCASCAG-3'; reverse primer, 5'-GGACTACNNGGGTATCTAAT-3'). The obtained PCR products were separated by electrophoresis in agarose gels $(2 \%, w / v)$, followed by purification by the Qiagen Gel Extraction Kit (Qiagen, Germany) and incorporation at equal concentrations (Qiu et al., 2018b). Lastly, the PCRpurified products were sequenced on an Illumina MiSeq 250 platform (Illumina, San Diego, CA, USA) according to the protocol described by Caporaso et al. (2010).

\section{Bioinformatic Analysis}

Using the QIIME program (http://qiime.sourceforge.net/), quality raw data were selected, in which sequences with an average length $<2000$ bp or an average quality score $<25$ were removed (Caporaso et al., 2010). In addition, chimera sequences were discarded using the UCHIME algorithm (Edgar et al., 2011). Using fast length adjustment of short reads (FLASH, v1.2.7), we assigned paired-end reads to each sample based on their unique barcodes and arranged them according to their overlapping sequences (Magoč and Salzberg, 2011). After trimming the barcode and sequence primers from the sequences, the valid sequences were classified into operational taxonomic units (OTUs) based on the sequence similarity with a 0.97 similarity threshold (Qiu et al., 2018a). Using the Naive Bayes classifier of the Ribosomal Database Project (RDP), the SILVA SSU database at the genus, family, order, class, and phylum levels was used to classify and annotate the taxonomic information for each representative OTU sequence (Quast et al., 2012; Yilmaz et al., 2014; Kalyuzhnaya et al., 2015). Using QIIME (Version 1.7.0), the alpha diversity was calculated to determine the diversity of the microbial community, and $\mathrm{R}$ software (Version 2.15.3) was used to analyze the beta diversity and compare the differences in the different treatment groups.

\section{RESULTS}

\section{Effect of $\boldsymbol{P}$. acidilactici Strains on Time to First Black-Stool Defecation}

The time to the first black-stool defecation was compared to evaluate the effect of bacteriocin-producing and non-bacteriocinproducing $P$. acidilactici strains on constipation in mice. As 
shown in Figure 1, the time to the first black-stool defecation of the different groups ranged from $3.92 \mathrm{~h}$ to $6.21 \mathrm{~h}$, with obvious differences. The time to the first black-stool defecation of mice in the normal group was $3.93 \mathrm{~h}$, which significantly increased to $6.21 \mathrm{~h}$ after treatment with loperamide hydrochloride $(P<0.05)$. However, the administration of phenolphthalein and four $P$. acidilactici strains was found to reduce the time to the first blackstool defecation in the mice. The shortest time to the first black-stool defecation occurred in the mice treated with phenolphthalein $(2.75 \mathrm{~h})$, which differed significantly from that of the normal group $(P<0.05)$. Although the time to the first black-stool defecation of mice in the $P$. acidilactici -treated groups was shorter than that of the negative control group, the differences were not significant $(P>0.05)$. Among the four strain-treatment groups, the mice treated with $P$. acidilactici NT17-3 had the shortest time to the first black-stool defecation $(5.38 \mathrm{~h})$, and the mice treated with $P$. acidilactici CCFM28 had the longest time $(6.10 \mathrm{~h})$. This time was $5.50 \mathrm{~h}$ for mice treated with the P. acidilactici CCFM18 and 102H8 strains. These results indicated that both the bacteriocin-producing and nonbacteriocin-producing $P$. acidilactici strains had limited therapeutic effects on constipation-related symptoms in mice. Interestingly, although there were no significant differences between the time to the first black-stool defecation in mice from the four $P$. acidilactici-treated groups, the nonbacteriocin-producing $P$. acidilactici strains seemed to contribute to a decreased time of first defecation.

\section{Effect of $P$. acidilactici Strains on the Gastrointestinal Transit Rate}

The gastrointestinal transit rate indicates the peristalsis or movement through the small intestine during digestion. According to the results shown in Figure 2, the administration of loperamide hydrochloride resulted in a $44.41 \%$ decrease in the gastrointestinal transit rate (from $68.90 \%$ to $38.30 \%$ ), which is significantly different from that of the mice in the normal control group $(P<0.05)$. This result indicated the successful establishment of a constipated model with the obstruction of normal peristalsis of the small intestine. The mice subsequently treated with phenolphthalein exhibited the highest gastrointestinal transit rate $(83 \%)$, which was significantly higher than those of the mice in other groups $(P<0.05)$. The administration of four $P$. acidilactici strains resulted in different increases in their gastrointestinal transit rates and improvements in their constipation-related symptoms. Mice treated with bacteriocinproducing $P$. acidilactici CCFM28 strains exhibited an increase from $38.30 \%$ to $55.10 \%$ in the gastrointestinal transit rate, which differed significantly from that of the negative control group $(P<$ 0.05). However, although the other three $P$. acidilactici strains increased the gastrointestinal transit rate in the mice, the differences between the $P$. acidilactici-treated and negative control groups were not significant $(P>0.05)$. We noted that the mice treated with the bacteriocin-producing $P$. acidilactici CCFM18 strain showed the lowest gastrointestinal transit rate (40.30\%). Furthermore, the recovery of the gastrointestinal transit

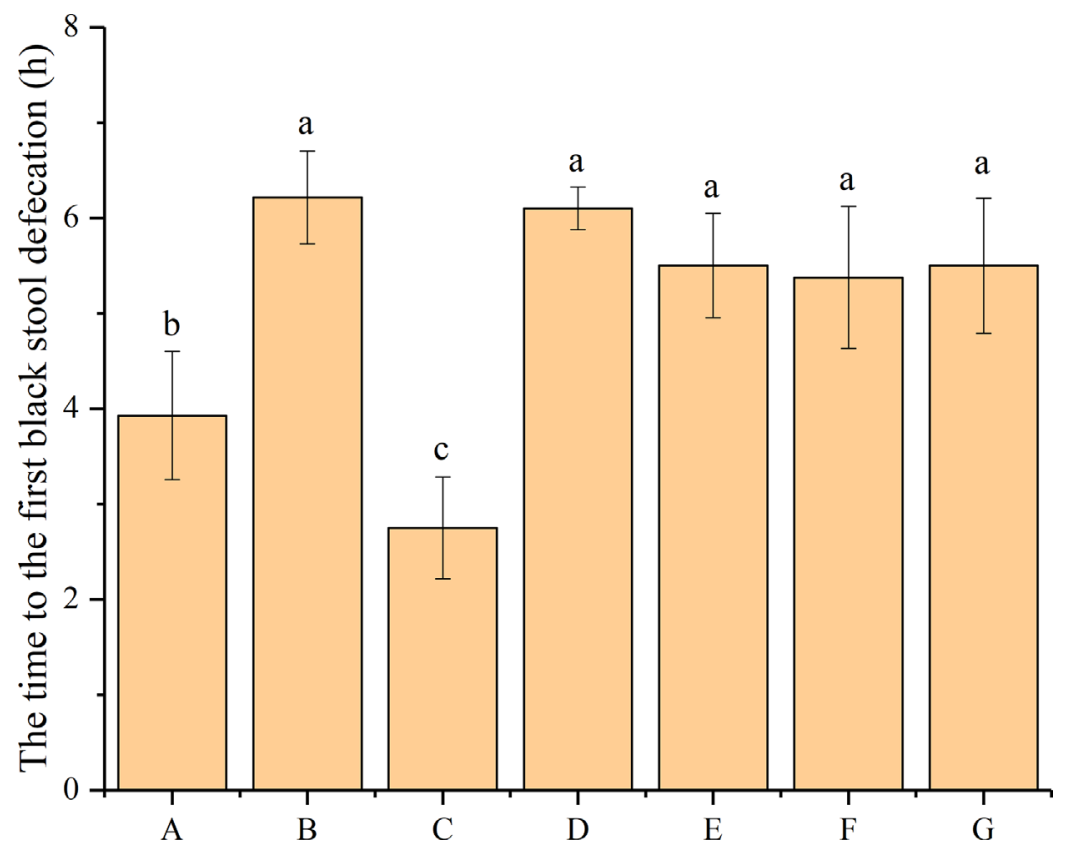

FIGURE 1 | Time to the first black-stool defecation in BALB/c mice after constipation had been induced by loperamide hydrochloride and different treatments were given ( $\mathrm{n}=10)$. (A) Normal control: no treatment; (B) negative control group: physiological saline; (C) positive control group: phenolphthalein (70 mg/kg body weight); (D) treatment group: P. acidilactici CCFM28 strain; (E) treatment group: P. acidilactici CCFM18 strain; (F) treatment group: P. acidilactici NT17-3 strain; (G) treatment group: $P$. acidilactici $102 \mathrm{H} 8$ strain. The letters $a, b$, and $c$ indicate significant differences $(P<0.05)$ as determined using Duncan's multiple range test. 


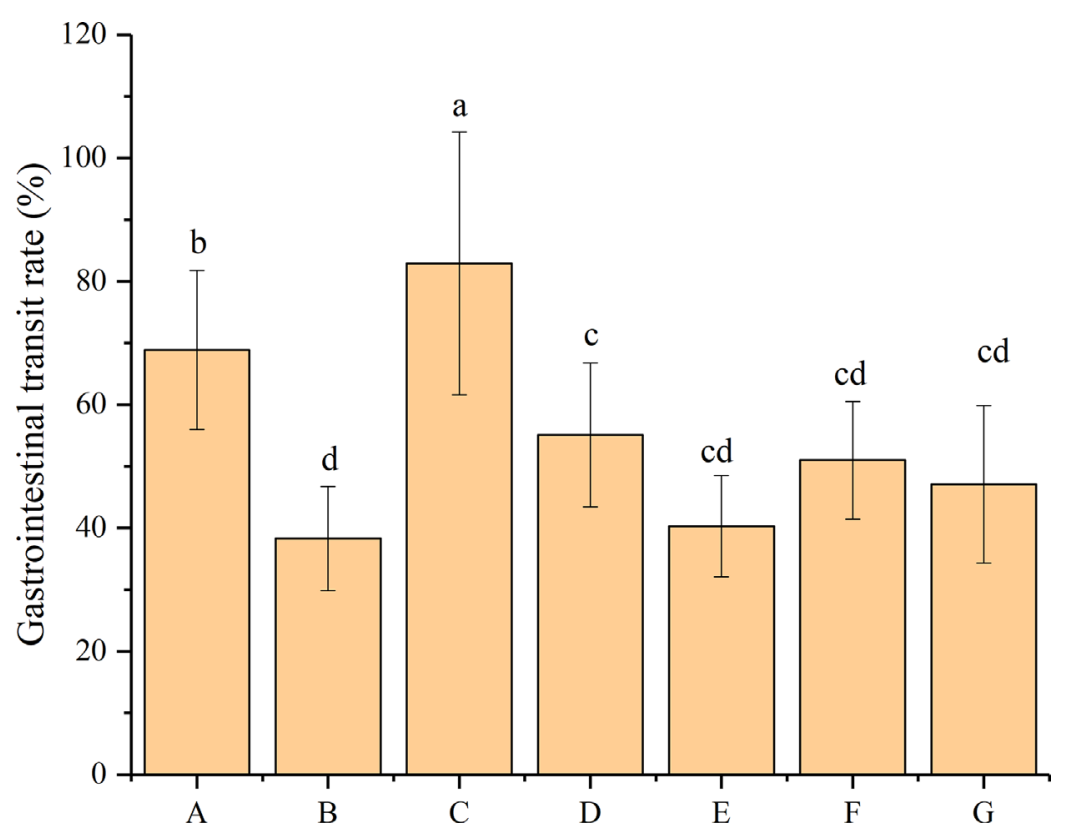

FIGURE 2 | Gastrointestinal transit rate of BALB/c mice after constipation induced by loperamide hydrochloride and administration of different treatments ( $\mathrm{n}=10$ ). (A) Normal control: no treatment; (B) negative control group: physiological saline; (C) positive control group: phenolphthalein (70 mg/kg body weight); (D) treatment group: $P$. acidilactici CCFM28 strain; (E) treatment group: $P$. acidilactici CCFM18 strain; (F) treatment group: $P$. acidilactici NT17-3 strain; (G) treatment group: $P$. acidilactici $102 \mathrm{H} 8$ strain. The letters $a, b, c$ and d indicate significant differences $(P<0.05)$ as determined using Duncan's multiple range test.

function of the mice treated with different $P$. acidilactici strains did not reach normal levels.

Based on the results obtained from the time to the first blackstool defecation and the gastrointestinal transit rate, the four $P$. acidilactici strains exhibited limited improvement in the constipation-related symptoms of the mice. The nonbacteriocin-producing $P$. acidilactici NT17-3 strain had the best effect on the time to the first black-stool defecation, and the bacteriocin-producing $P$. acidilactici CCFM28 strain significantly improved the small-intestine peristalsis.

\section{Effect of $\boldsymbol{P}$. acidilactici Strains on Gastrointestinal Regulatory Peptides in Serum of Constipated Mice}

Gastrointestinal regulatory peptides related to constipation, such as SP, MTL, Gas, ET, SS, and VIP, play crucial roles in the regulation of gastrointestinal motility (Schulz et al., 2004). Among them, three peptides (SP, MTL, and Gas) are known to be excitatory neurotransmitters, with the other three peptides (SS, VIP, and ET) being inhibitory neurotransmitters. SP, a neuropeptide that is widely distributed in fine nerve fibers, is reported to enhance the contraction of gastrointestinal smooth muscles and promote gastrointestinal motility (Du et al., 2003). Similarly, gastrointestinal motility is improved by MTL and Gas via the regulation of muscular movement and the secretion and transportation of digestive juices. In contrast, SS and VIP are considered to be regulatory inhibitory peptides that inhibit the secretion of several digestive enzymes and movement of intestinal muscles (Lucey, 1986).
As shown in Figure 3, the excitatory neurotransmitters (SP, MTL, and Gas) in the mice treated with loperamide hydrochloride exhibit a downward trend, whereas the inhibitory neurotransmitters (SS, VIP, and ET) were significantly upregulated $(P<0.05)$. This indicated that the ingestion of loperamide hydrochloride resulted in decreased gastrointestinal motility and mucus secretion. The phenolphthalein and four $P$. acidilactici strains effectively regulated the levels of excitatory and inhibitory neurotransmitters, including the upregulation of SP, MTL, and Gas, and the downregulation of SS, VIP, and ET. Specifically, the MTL level was significantly increased by $16.18-27.53 \%$ following the administration of $P$. acidilactici strains $(P<0.05)$, compared with the negative control group. Regarding SP and Gas, treatment with the four $P$. acidilactici strains resulted in 4.82$17.65 \%$ and $1.73-23.89 \%$ increases, respectively. Moreover, only the non-bacteriocin-producing $P$. acidilactici NT17-3 strain caused a significant increase in Gas $(P<0.05)$, whereas only the increase in SP was not significant in the mice treated with the non-bacteriocin-producing $P$. acidilactici $102 \mathrm{H} 8$ strain $(P<0.05)$.

In contrast, all three inhibitory neurotransmitters (SS, VIP, and ET) were downregulated after the introduction of different $P$. acidilactici strains, with the degree of reduction showing a significant difference $(P<0.05)$. The SS level decreased by $22.57-$ $31.88 \%$ following treatment with the $P$. acidilactici strains, with the ET level decreasing by $32.19-41.78 \%$ and the VIP level decreasing by $27.67-35.35 \%$. We noted that the $P$. acidilactici strains showed greater regulatory effects on the inhibitory 

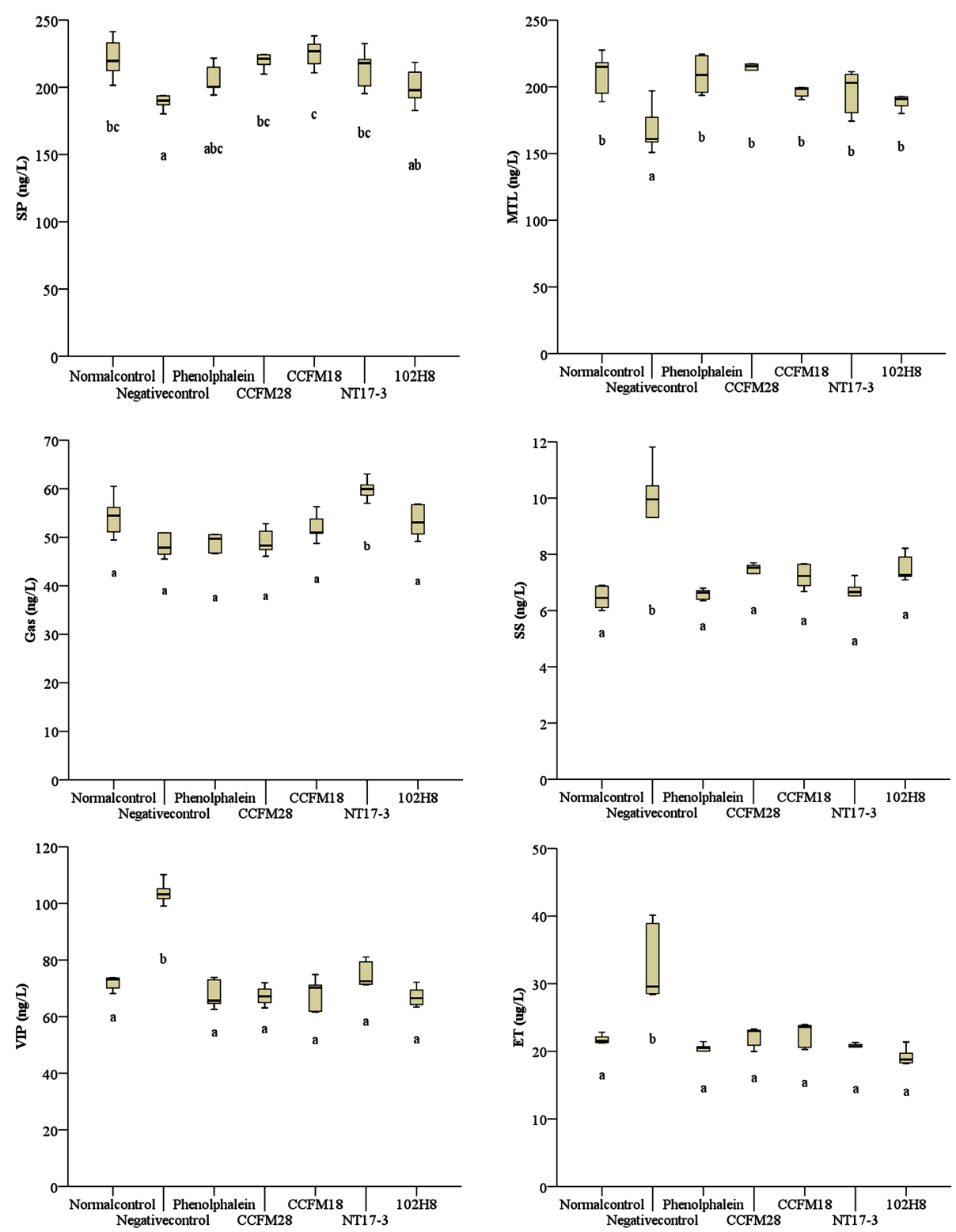

FIGURE 3 | Levels of gastrointestinal regulatory peptides in the serum of BALB/c mice after constipation had been induced by loperamide hydrochloride and different treatments were given $(n=10)$. SP: substance P; MTL: motilin; Gas: gastrin; SS: somatostatin; VIP: vasoactive intestinal peptide; ET: endothelin. (A) Normal control: no treatment; (B) negative control group: physiological saline; (C) positive control group: phenolphthalein (70 mg/kg body weight); (D) treatment group: P. acidilactici CCFM28 strain; (E) treatment group: P. acidilactici CCFM18 strain; (F) treatment group: P. acidilactici NT17-3 strain; (G) treatment group: P. acidilactici $102 \mathrm{H} 8$ strain. The letters $\mathrm{a}, \mathrm{b}$, and $\mathrm{c}$ indicate significant differences $(\mathrm{P}<0.05)$ as determined using Duncan's multiple range test.

neurotransmitters than on the excitatory neurotransmitters. Furthermore, significant differences between different $P$. acidilactici strains occurred only in Gas between the $P$. acidilactici NT17-3 strain and three other strains, and in SP between the P. acidilactici NT17-3 and 102H8 strains.

\section{Sequence and OTU Statistical Analysis}

Using the Illumina MiSeq sequencing platform, a total of 929,810 high-quality $16 \mathrm{~S}$ rRNA gene sequences were generated from 70 fecal samples. For each sample, the average sequence read was
13,283 after sequence assembly and quality control. At a $97 \%$ confidence threshold, all of the sequences were clustered with the representative sequences, and the number of OTUs ranged from 8,639 to 63,803 .

\section{Effect of $P$. acidilactici Strains on the Alpha and Beta Diversities of Fecal Microbiota}

The alpha diversity index reflects the abundance and diversity of the microbial community in an individual sample, including 
TABLE 1 | Alpha diversity indexes of microbial communities.

\begin{tabular}{|c|c|c|c|c|c|}
\hline Groups & Coverage & Chao1 index & Observed species & Shannon index & PD_whole_tree \\
\hline Normal control group & $0.9151^{b}$ & $5076.11^{d}$ & $1079.0^{d}$ & $5.60^{a}$ & $72.93^{\mathrm{C}}$ \\
\hline Negative control group & $0.9292^{\mathrm{ab}}$ & $10470.77^{b c}$ & $2432.2^{b c}$ & $5.94^{a}$ & $124.50^{b}$ \\
\hline Phenolphthalein & $0.9287^{a b}$ & $7599.74^{\mathrm{cd}}$ & $1668.5^{\mathrm{cd}}$ & $5.32^{\mathrm{a}}$ & $94.31^{\mathrm{bc}}$ \\
\hline CCFM28 & $0.9455^{\mathrm{a}}$ & $16291.02^{\mathrm{a}}$ & $3876.5^{\mathrm{a}}$ & $5.73^{a}$ & $179.68^{a}$ \\
\hline CCFM18 & $0.9196^{b}$ & $4924.21^{d}$ & $1165.5^{d}$ & $5.60^{a}$ & $78.30^{\circ}$ \\
\hline NT17-3 & $0.9447^{\mathrm{a}}$ & $12021.24^{b}$ & $2781.3^{b}$ & $5.72^{a}$ & $137.92^{b}$ \\
\hline $102 \mathrm{H} 8$ & $0.9318^{a b}$ & $8989.47^{\text {bcd }}$ & $1970.6^{\mathrm{bcd}}$ & $5.56^{a}$ & $105.32^{b c}$ \\
\hline
\end{tabular}

Different small letters in the same column means a significant difference $(P<0.05)$.

coverage, the chaol index, observed species, Shannon index, and PD_whole_tree. As shown in Table 1, the five index values of the normal group averaged $0.9151,5076.11,1079.0,5.60$, and 72.93 , respectively. The administration of phenolphthalein in the constipated mice led to great changes in these indexes to $0.9287,7599.74,1668.5,5.32$, and 94.31 , respectively. For the $P$. acidilactici-treated groups, the application of different strains resulted in an increasing trend of the alpha diversity of the microbial community in the fecal microbiota. Specifically, although there was no significant difference in the Shannon indices of the seven groups, the chaol and observed species indices of the mice treated with the bacteriocin-producing $P$. acidilactici CCFM28 strain were significantly higher than those of the other groups. This result indicated that this strain significantly increased the microbial diversity of the intestinal flora of the mice $(P<0.05)$.

Figure 4 showed an unweighted uniFrac matrix of the beta diversities in the gut microbiota of mice treated with the four $P$. acidilactici strains at the genus level, which revealed differences and similarities among the samples. The distribution maps of the first two principal components represented $11.43 \%$ (PC1) and $4.5 \%$ (PC2) of the accumulative variance contributions, for a
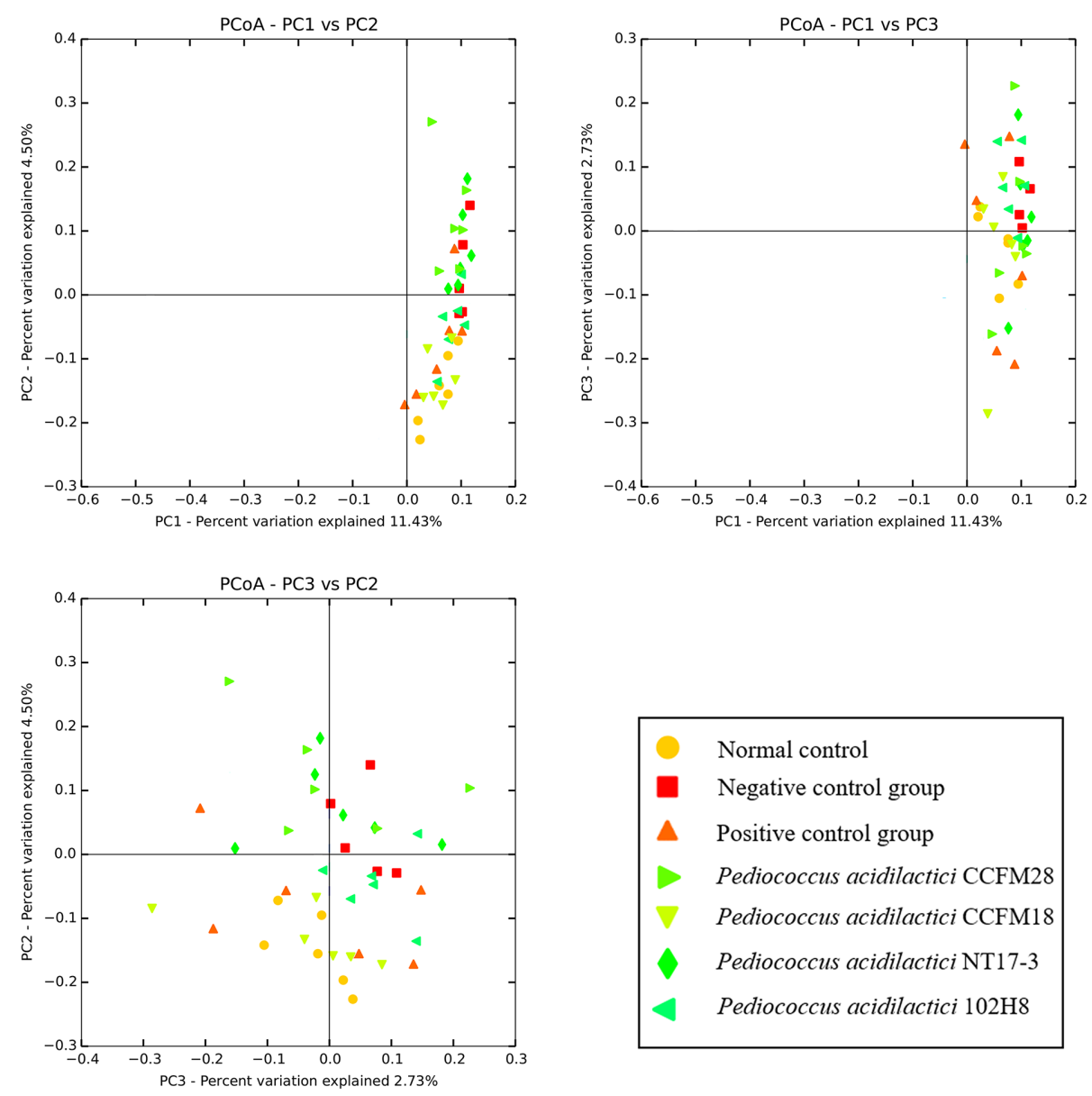

Normal control

Negative control group

Positive control group

Pediococcus acidilactici CCFM28

Pediococcus acidilactici CCFM18

Pediococcus acidilactici NT17-3

Pediococcus acidilactici $102 \mathrm{H} 8$

FIGURE 4 | Principal component analysis of microbial communities in mice feces. 
total of $15.9 \%$. The microbial communities of the mice treated with phenolphthalein and the mice in the normal control group were clustered together, which indicated that treatment with phenolphthalein was effective in adjusting the structure of the intestinal flora in constipated mice to a normal level. The microbial communities of mice treated with the $P$. acidilactici CCFM18 and 102H8 strains and the mice in the normal control group also overlapped to some extent, which indicated a high degree of similarity in their microbial communities. However, the microbial communities of the mice treated with the $P$. acidilactici CCFM28 and NT17-3 strains could be readily distinguished from those of the normal control group, which meant that the application of $P$. acidilactici NT17-3 clearly changed the structure of the gut microbiota in the mice.

\section{Effect of $P$. acidilactici Strains on the Composition of Fecal Microbiota at the Phylum Level}

Figure 5 showed the distributions and variances in the bacterial community compositions at the phylum level in normal and constipated mice given different treatments. The fecal flora of normal mice mainly consisted of Firmicutes (68.98\%), Proteobacteria (15.00\%), and Bacteroidetes (9.23\%). Other phyla were also detected, but they were low in abundance ( $<1 \%)$, including Tenericutes, Verrucomicrobia, and Cyanobacteria. After treatment with loperamide hydrochloride, great changes occurred in the fecal microbiomes of mice in the negative control group. The relative abundance of Firmicutes was significantly reduced by $29.21 \%$ (from $68.98 \%$ to $53.54 \%$ ), whereas the relative abundances of Proteobacteria and Bacteroidetes were significantly increased by $70.33 \%$ and $71.55 \%$, respectively $(P<0.05)$. The administration of the four $P$. acidilactici strains effectively reversed this change, where the relative abundances of Firmicutes and Actinobacteria were upregulated, and those of Proteobacteria and Bacteroidetes were downregulated. The Firmicutes included some intestinal probiotics (i.e., Lactobacillus and Lactococcus), and the Proteobacteria contained many pathogens (including Salmonella and Escherichia coli). Therefore, changes derived from the interference of the $P$. acidilactici strains indicated the improvement in the intestinal environment.

The distribution and relative abundance of the bacterial community at the phylum level of the mice in the $P$. acidilactici CCFM28-treated group were closest to those of mice in the normal group. The relative abundance of Firmicutes increased from $53.54 \%$ to $63.53 \%$, but the Proteobacteria and Bacteroidetes decreased from 25.54\% and $15.84 \%$ to $15.38 \%$ and $11.74 \%$, respectively. The microbial community of the mice in the $P$. acidilactici CCFM18-treated group exhibited changes similar to those of the $P$. acidilactici CCFM28-treated group, which indicated that it also effectively regulated the composition of the mouse intestinal flora. An increase of $10.52 \%$ in the relative abundance of Firmicutes and decreases of $16.00 \%$ and $38.78 \%$ in the respective levels of
A
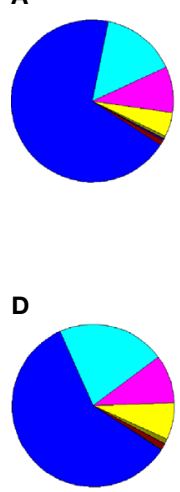

G

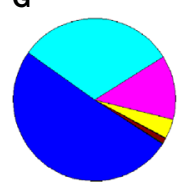

B

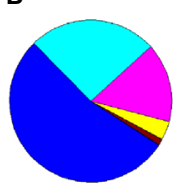

E

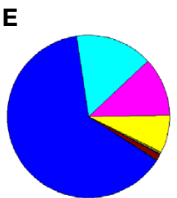

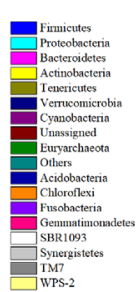

C
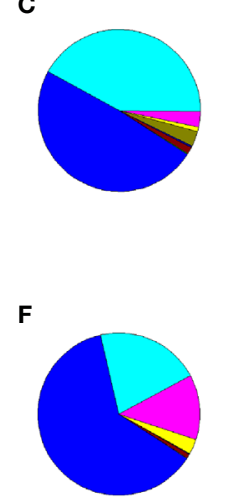
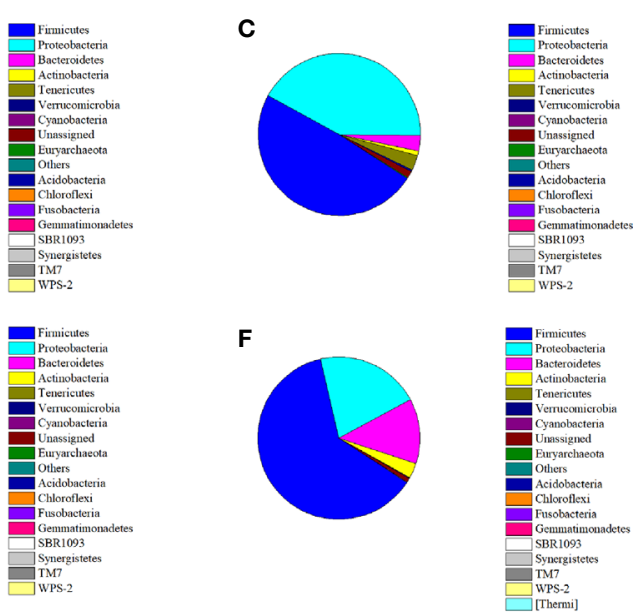

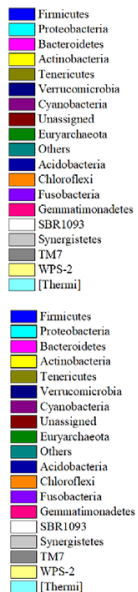

FIGURE 5 | Relative abundance of main phyla in the different mice groups after constipation induced by loperamide hydrochloride and the administration of different treatments $(n=10)$. (A) Normal control: no treatment; (B) negative control group: physiological saline; (C) positive control group: phenolphthalein (70 mg/kg body weight); (D) treatment group: P. acidilactici CCFM28 strain; (E) treatment group: P. acidilactici CCFM18 strain; (F) treatment group: P. acidilactici NT17-3 strain; (G) treatment group: P. acidilactici $102 \mathrm{H} 8$ strain. 
Proteobacteria and Bacteroidetes occurred in the mice treated with the $P$. acidilactici CCFM18 strain. In the non-bacteriocinproducing $P$. acidilactici-treated groups, the regulation of intestinal flora by the $P$. acidilactici NT17-3 strain was similar to that of the bacteriocin-producing $P$. acidilactici CCFM28 strain, including an increase of $16.10 \%$ in Firmicutes and decreases of $18.39 \%$ and $18.66 \%$ in Proteobacteria and Bacteroidetes, respectively. Although the phyla with high abundance in the $P$. acidilactici $102 \mathrm{H} 8$-treated group were almost identical to those of the other groups, there was no obvious change in the relative abundance of Firmicutes. Therefore, we could conclude that all four P. acidilactici strains reduced the relative abundances of Proteobacteria and Bacteroidetes and increased the level of Firmicutes, but $P$. acidilactici CCFM28 was most effective.

The observed changes in the intestinal flora were similar to the results reported by Wang et al. (2017). They found that the intervention of Bifidobacterium adolescentis CCFM 669 and 667 strains increased the ratio of relative abundance of Firmicutes to Bacteroidetes at the phylum level in constipated BALB/c mice (Wang et al., 2017). In our study, the administration of loperamide hydrochloride in mice was found to disturb the ecological balance of intestinal flora, which was mainly manifested in the reduced levels of Firmicutes and the increased levels of Proteobacteria and Bacteroidetes (including many pathogens or enteropathogenic bacteria). However, the disruption of the microbial structure was moderated by the introduction of four $P$. acidilactici strains, which might generate a space-occupying competition or the production of some metabolites (such as bacteriocins and SCFAs).

\section{Effect of $P$. acidilactici Strains on the Composition of Fecal Microbiota at the Genus Level}

At the genus level, a total of 675 bacterial genera were classified, of which 22 genera were found to have relative abundances greater than 1\%. Figure 6 showed the relative abundances of main genera in the different mice groups. The relative abundance of Blautia was greatest in the mice of the normal control group (24.10\%), followed by several genera from Clostridiaceae (16.01\%), Enterobacteriaceae (10.53\%), and Ruminococcaceae (6.30\%). Bacteroides and Bifidobacterium also accounted for large proportions (4.94\% and $3.43 \%$, respectively). Treatment with loperamide hydrochloride resulted in a major change in the relative abundance of the bacterial community in mice. The proportions of Blautia, Bifidobacterium, Lactobacillus, and some genera from Clostridiaceae and Ruminococcaceae dropped significantly, whereas Bacteroides, Klebsiella, and certain genera from Enterobacteriaceae were obviously increased.

After the administration of $P$. acidilactici strains, the relative abundances of Bifidobacterium and Lactobacillus increased significantly $(P<0.05)$, i.e., $4-21$-fold and $1-37$-fold, respectively, compared with those in the negative control group. Bifidobacterium and Lactobacillus are considered to be

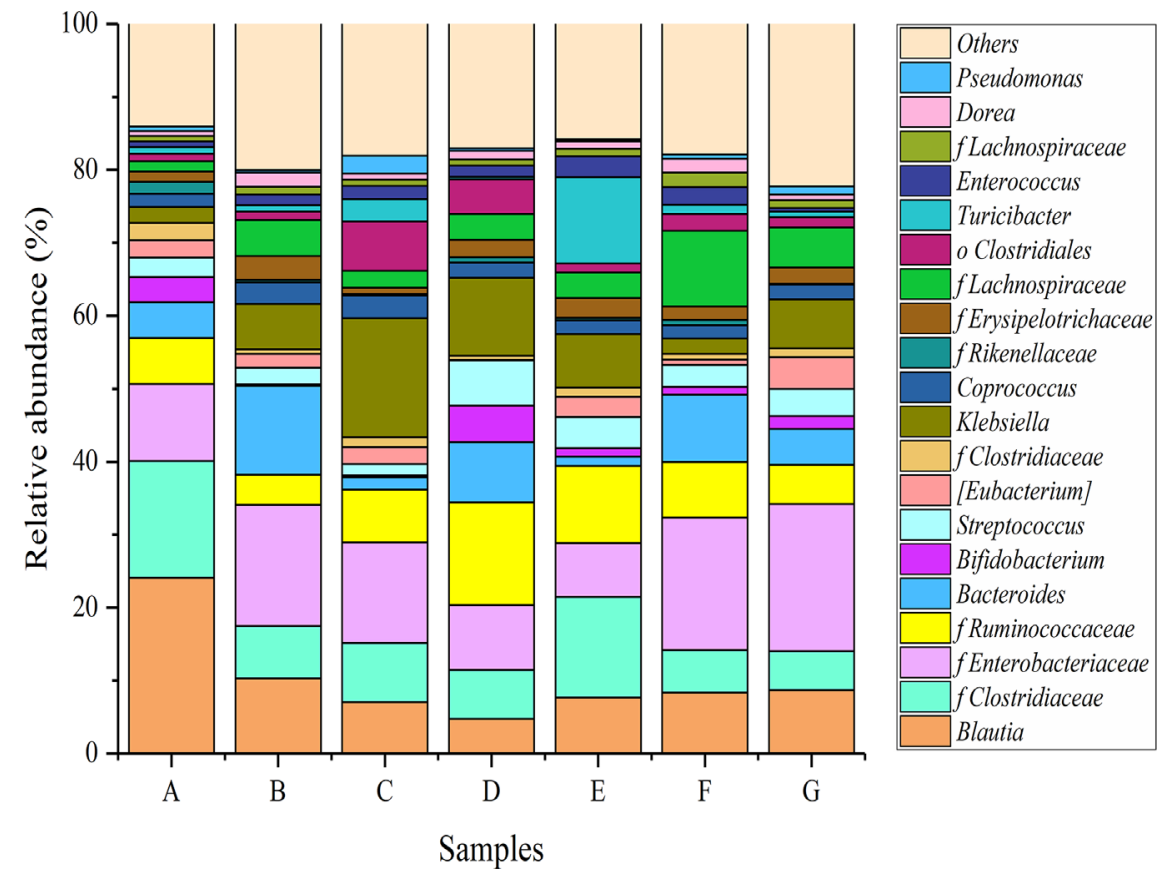

FIGURE 6 | Relative abundances of main genera in the different mice groups after constipation had been induced by loperamide hydrochloride and different treatments were administered $(\mathrm{n}=10)$. (A) Normal control: no treatment; (B) negative control group: physiological saline; (C) positive control group: phenolphthalein (70 mg/kg body weight); (D) treatment group: $P$. acidilactici CCFM28 strain; (E) treatment group: $P$. acidilactici CCFM18 strain; (F) treatment group: $P$. acidilactici NT17-3 strain; (G) treatment group: P. acidilactici $102 \mathrm{H} 8$ strain. 
important intestinal beneficial microorganisms that can regulate gastrointestinal function and enhance human immunity (Meile et al., 2008). A significant decrease in the relative abundance of Bacteroides was also observed following treatment with the four $P$. acidilactici strains, with a 24.13$89.34 \%$ decrease compared with the negative control group. The authors of another study reported that Bacteroides inhabited the human intestines and might cause endogenous infections when the body's immune function was disrupted or the flora was unbalanced due to the use of antibiotics. The relative abundances of Blautia and several genera from Erysipelotrichaceae and Lachnospiraceae further decreased when the $P$. acidilactici strains were administered. Enterobacteriaceae contains a variety of intestinal pathogens, such as Escherichia coli, Salmonella, and Shigella, which are closely associated with intestinal diseases (including bacterial diarrhea, gastroenteritis, and dysentery) (Coburn et al., 2007).

Compared with the negative control group, treatment with the bacteriocin-producing P. acidilactici CCFM28 and CCFM18 strains significantly decreased the relative abundance of the genera from Enterobacteriaceae, especially the $P$. acidilactici CCFM28 strain. However, non-bacteriocin-producing $P$. acidilactici strains did not achieve this effect, which might be due to the absence of bacteriocin.

\section{DISCUSSION}

Constipation is a common gastrointestinal functional disorder worldwide and seriously affects the quality of life of patients. The effectiveness of probiotics in relieving functional constipation is reported to be due to their regulation of aberrant gut motility via metabolites such as SCFAs (Dimidi et al., 2017) and tryptamine (Bhattarai et al., 2018). However, there have been no reports regarding differences in the degree of alleviation provided by different $P$. acidilactici strains (bacteriocin-producing and nonbacteriocin-producing) of constipation or the resulting changes in the fecal flora in mice.

The results of this study indicated that the administration of four $P$. acidilactici strains produced some improvement in constipation-related symptoms (based on the time to the first black-stool defecation and gastrointestinal transit rate), but the difference was not significant $(P>0.05)$. However, there were significant differences in the compositions and relative abundances of the intestinal microbiota in different groups. At the phylum level, the relative abundance of Firmicutes significantly increased, but those of Bacteroidetes and Proteobacteria decreased after the administration of four $P$. acidilactici strains for $14 \mathrm{~d}$. Furthermore, the levels of Bacteroides and several genera from Enterobacteriaceae were significantly decreased, whereas Bifidobacterium and Lactobacillus were upregulated following the provision of bacteriocin-producing $P$. acidilactici CCFM18 and CCFM28 strains in the diet. Therefore, although constipation-related symptoms were only marginally alleviated, the four $P$. acidilactici strains were found to regulate the intestinal flora and provide a potential health benefit to the host. Bacteriocinproducing P. acidilactici strains, in particular, were more effective in restoring the intestinal flora to normal levels.

Bacteriocins, as ribosomally synthesized peptides or proteins with potential antimicrobial activity, are produced by many bacterial species (Gálvez et al., 2007). Bacteriocin production may play an important role in the competition within complex microbial communities or in positively influencing the health of the host. The factors that contribute to its probiotic functionality may derive from several mechanisms within the gastrointestinal tract. For example, some bacteriocins have been considered to be colonizing peptides that can facilitate the introduction of a probiotic into an existing niche or compete with the resident microbiota (Riley and Wertz, 2002). Bacteriocins may also function as antimicrobial peptides that can inhibit or eliminate pathogens (Majeed et al., 2011). Some signaling peptides may signal the immune cells of the host or other bacteria (Di Cagno et al., 2007; Gobbetti et al., 2007). These mechanisms may act alone or in combination in the gastrointestinal tract of mice supplemented with bacteriocinproducing $P$. acidilactici CCFM18 and CCFM28 strains. However, additional research is required to clarify how those two strains affect intestinal flora.

The administration of $P$. acidilactici strains resulted in significant increases in the relative abundances of Bifidobacterium and Lactobacillus (4-21-fold and 1-37-fold, respectively), compared with that in the negative control group $(P<0.05)$. Furthermore, administration of the bacteriocinproducing $P$. acidilactici CCFM28 and CCFM18 strains significantly decreased the relative abundance of the genera from Enterobacteriaceae. The increase in beneficial bacteria and decrease in harmful bacteria are closely related to improvement of the constipation symptoms. For example, the SCFAs produced by Bifidobacterium and Lactobacillus were found to relieve symptoms of constipation by stimulating the absorption of water and electrolytes, promoting the proliferation of epithelial cells, improving gastrointestinal motility, and increasing mesenteric blood flow (Ríos-Covián et al., 2016). Furthermore, in this study, the four $P$. acidilactici strains were found to cause upregulation of excitatory neurotransmitters (SP, MTL, and Gas) and the downregulation of inhibitory neurotransmitters (SS, VIP, and ET). Therefore, changes in the gut bacteria can also alleviate constipation by influencing the levels of gastrointestinal hormones, which counteract the symptoms of constipation via the enhancement of intestinal peristalsis and the transport of contents. These findings agree well with the results of Wang et al. (2017).

Extensive use of antibiotics might increase the relative abundance of Clostridium, leading to antibiotic-related diarrhea and pseudomembranous colitis (Kim et al., 1981). Treatment with bacteriocin-producing $P$. acidilactici CCFM18 strain resulted in a $6.34 \%$ decrease in the relative abundance of the genera from Clostridiaceae, thereby reducing the likely incidence of inflammatory diseases. Therefore, the decrease in the relative abundance of harmful intestinal microorganisms and the increase in probiotics indicated that the administration of bacteriocin- 
producing and non-bacteriocin-producing $P$. acidilactici strains had the potential to regulate the intestinal flora, maintain human health, and replace antibiotic drugs, with the bacteriocin-producing $P$. acidilactici CCFM28 strain being especially effective.

\section{CONCLUSIONS}

In conclusion, we investigated the effects of the administration of bacteriocin-producing and non-bacteriocin-producing Pediococcus acidilactici strains on constipation symptoms, gastrointestinal regulatory peptides and gut microbiota in $\mathrm{BALB} / \mathrm{c}$ mice. The treatment of four $P$. acidilactici strains could limitedly improve the first black-stool defecation and the gastrointestinal transit rate in constipated mice $(P>0.05)$, significantly regulated the level of most gastrointestinal regulatory peptides in the serum $(P<0.05)$. Furthermore, the composition and abundance of intestinal microbiota in different groups were greatly changed by four $P$. acidilactici strains. The intervention of four $P$. acidilactici strains on constipated mice effectively reversed the change in the gut flora caused by the treatment of loperamide hydrochloride and restored their composition and relative abundance. Therefore, although constipation-related symptoms were alleviated to only a limited degree, the administration of four $P$. acidilactici strains effectively regulated the gut flora and provided a potential health benefit to the host, especially the bacteriocin-producing $P$. acidilactici strains. Future work should be directed towards the characterization of bacteriocins produced by $P$. acidilactici strains, and the exploration of the interaction between bacteriocins and the intestinal flora in mice.

\section{DATA AVAILABILITY STATEMENT}

The raw data supporting the conclusions of this article will be made available by the authors, without undue reservation. The

\section{REFERENCES}

Battish, R., Cao, G. Y., Lynn, R. B., Chakder, S., and Rattan, S. (2000). Heme oxygenase-2 distribution in anorectum: colocalization with neuronal nitric oxide synthase. Am. J. Physiol-Gastr. L. 278, G148-G155. doi: 10.1152/ ajpgi.2000.278.1.G148

Bezkorovainy, A. (2001). Probiotics: determinants of survival and growth in the gut. Am. J. Clin. Nutr. 73, 399s-405s. doi: 10.1093/ajcn/73.2.399s

Bharucha, A. E., Wouters, M. M., and Tack, J. (2017). Existing and emerging therapies for managing constipation and diarrhea. Curr. Opin. Pharmacol. 37, 158-166. doi: 10.1016/j.coph.2017.10.015

Bharucha, A. E. (2007). Constipation. Best Pract. Res. Cl. Ga. 21, 709-731. doi: 10.1016/B978-1-4377-1793-8.00098-4

Bhattarai, Y., Williams, B. B., Battaglioli, E. J., Whitaker, W. R., Till, L., Grover, M., et al. (2018). Gut microbiota-produced tryptamine activates an epithelial Gprotein-coupled receptor to increase colonic secretion. Cell Host Microbe 23, 775-785. doi: 10.1016/j.chom.2018.05.004

Bu, L. N., Chang, M. H., Ni, Y. H., Chen, H. L., and Cheng, C. C. (2007). Lactobacillus casei rhamnosus Lcr35 in children with chronic constipation. Pediatr. Int. 49, 485-490. doi: 10.1111/j.1442-200X.2007.02397.x

Caporaso, J. G., Kuczynski, J., Stombaugh, J., Bittinger, K., Bushman, F. D., Costello, E. $\mathrm{K}$., et al. (2010). QIIME allows analysis of high-throughput community sequencing data. Nat. Methods 7, 335-336. doi: 10.1038/nmeth.f.303 data presented in the study are deposited in the SRA repository, accession number (PRJNA705063).

\section{ETHICS STATEMENT}

The animal study was reviewed and approved by The Ethics Committee of Jiangnan University, China (JN. No 2017121920180129 [169]), and this experiment was conducted according to the European Community guidelines (Directive 2010/63/EU).

\section{AUTHOR CONTRIBUTIONS}

YQ, ZQ, QZ, and WC were responsible for conceptualization, methodology and software. YQ, ZQ, FT, LY, JZ, and HZ were responsible for data curation, writing-original draft preparation, visualization, and investigation. QZ and WC were responsible for supervision and validation. $\mathrm{YQ}, \mathrm{ZQ}, \mathrm{QZ}$, and $\mathrm{WC}$ were responsible for writing-reviewing and editing. All authors contributed to the article and approved the submitted version.

\section{FUNDING}

This work was supported by the National Natural Science Foundation of China Program (31820103010, 31871773, and U1903205); the Key Scientific and Technological Research Projects in the Key Areas of the Xinjiang Production and Construction Corps (2018AB010); National First Class Discipline Program of Food Science and Technology (JUFSTR20180102); the BBSRC Newton Fund Joint Centre Award; and Collaborative Innovation Center of Food Safety and Quality Control in Jiangsu Province.

Chelliah, R., Saravanakumar, K., Daliri, E. B. M., Kim, J. H., Lee, J. K., Jo, H. Y., et al. (2020). Unveiling the potentials of bacteriocin (Pediocin L50) from Pediococcus acidilactici with antagonist spectrum in a Caenorhabditis elegans model. Int. J. Biol. Macromol. 143, 555-572. doi: 10.1016/j.ijbiomac.2019.10.196

Coburn, B., Grassl, G. A., and Finlay, B. B. (2007). Salmonella, the host and disease: a brief review. Immunol. Cell Biol. 85, 112-118. doi: 10.1038/sj.icb.7100007

Corr, S. C., Li, Y., Riedel, C. U., O’Toole, P. W., Hill, C., and Gahan, C. G. (2007). Bacteriocin production as a mechanism for the antiinfective activity of Lactobacillus salivarius UCC118. Proc. Natl. Acad. Sci. U.S.A 104, 76177621. doi: 10.1073/pnas.0700440104

Di Cagno, R., De Angelis, M., Limitone, A., Minervini, F., Simonetti, M. C., Buchin, S., et al. (2007). Cell-cell communication in sourdough lactic acid bacteria: a proteomic study in Lactobacillus sanfranciscensis CB1. Proteomics 7, 2430-2446. doi: 10.1002/pmic.200700143

Dimidi, E., Christodoulides, S., Scott, S. M., and Whelan, K. (2017). Mechanisms of action of probiotics and the gastrointestinal microbiota on gut motility and constipation. Adv. Nutr. 8, 484-494. doi: 10.3945/an.116.014407

Dimidi, E., Scott, S. M., and Whelan, K. (2020). Probiotics and constipation: Mechanisms of action, evidence for effectiveness and utilisation by patients and healthcare professionals. P. Nutr. Soc 79, 147-157. doi: 10.1017/S0029665119000934

Du, L. F., Zhan, S. Q., and Guo, X. K. (2003). The relationship between substance $\mathrm{P}$, vasoactive intestinal peptide and abnormal gastrointestinal transit. J. Xi'an Jiaotong Med. Univ. 24, 363-365. 
Edgar, R. C., Haas, B. J., Clemente, J. C., Quince, C., and Knight, R. (2011). UCHIME improves sensitivity and speed of chimera detection. Bioinformatics 27, 2194-2200. doi: 10.1093/bioinformatics/btr381

Eskesen, D., Jespersen, L., Michelsen, B., Whorwell, P. J., Müller-Lissner, S., and Morberg, C. M. (2015). Effect of the probiotic strain Bifidobacterium animalis subsp. lactis, BB-12 ${ }^{\circledR}$, on defecation frequency in healthy subjects with low defecation frequency and abdominal discomfort: a randomised, double-blind, placebo-controlled, parallel-group trial. Brit. J. Nutr. 114, 1638-1646. doi: 10.1017/S0007114515003347

Gálvez, A., Abriouel, H., López, R. L., and Omar, N. B. (2007). Bacteriocin-based strategies for food biopreservation. Int. J. Food Microbiol. 120, 51-70. doi: 10.1016/j.ijfoodmicro.2007.06.001

Gobbetti, M., De Angelis, M., Di Cagno, R., Minervini, F., and Limitone, A. (2007). Cell-cell communication in food related bacteria. Int. J. Food Microbiol. 120, 34-45. doi: 10.1016/j.ijfoodmicro.2007.06.012

Gonzalez, C. F., and Kunka, B. S. (1987). Plasmid-associated bacteriocin production and sucrose fermentation in Pediococcus acidilactici. Appl. Environ. Microb. 53, 2534-2538. doi: 10.1002/bit.260300516

Heeney, D. D., Zhai, Z., Bendiks, Z., Barouei, J., Martinic, A., Slupsky, C., et al. (2019). Lactobacillus plantarum bacteriocin is associated with intestinal and systemic improvements in diet-induced obese mice and maintains epithelial barrier integrity in vitro. Gut Microbes 10, 382-397. doi: 10.1080/ 19490976.2018.1534513

Kalyuzhnaya, M. G., Yang, S., Beck, D. A., and Chistoserdova, L. (2015). "Systems biology tools for methylotrophs," in Hydrocarbon and Lipid Microbiology Protocols (Berlin, Heidelberg: Springer), 97-118.

Kim, K. H., Fekety, R., Batts, D. H., Brown, D., Cudmore, M., Silva, J.Jr, et al. (1981). Isolation of Clostridium difficile from the environment and contacts of patients with antibiotic-associated colitis. J. Infect. Dis. 143, 42-50. doi: 10.1093/infdis/143.1.42

Kommineni, S., Bretl, D. J., Lam, V., Chakraborty, R., Hayward, M., Simpson, P., et al. (2015). Bacteriocin production augments niche competition by enterococci in the mammalian gastrointestinal tract. Nature 526, 719-722. doi: 10.1038/nature15524

Lee, H. Y., Kim, J. H., Jeung, H. W., Lee, C. U., Kim, D. S., Li, B., et al. (2012). Effects of Ficus carica paste on loperamide-induced constipation in rats. Food Chem. Toxicol. 50, 895-902. doi: 10.1016/j.fct.2011.12.001

Lin, Z. H., Wu, J. X., Xiao, Z. D., Wu, B. H., Han, Z. P., Yi, X. M., et al. (2005). The curative effect of aloe on constipation and its primary mechanism. Guangdong Med. J. 025, 1336-1338. doi: 10.13820/j.cnki.gdyx.2005.10.016

Lucey, M. R. (1986). Endogenous somatostatin and the gut. Gut 27:457. doi: 10.1136/gut.27.4.457

Luthra, P., Burr, N. E., Brenner, D. M., and Ford, A. C. (2019). Efficacy of pharmacological therapies for the treatment of opioid-induced constipation: systematic review and network meta-analysis. Gut 68, 434-444. doi: 10.1136/ gutjnl-2018-316001

Magoč, T., and Salzberg, S. L. (2011). FLASH: fast length adjustment of short reads to improve genome assemblies. Bioinformatics 27, 2957-2963. doi: 10.1093/ bioinformatics/btr507

Majeed, H., Gillor, O., Kerr, B., and Riley, M. A. (2011). Competitive interactions in Escherichia coli populations: the role of bacteriocins. ISME J. 5, 71-81. doi: $10.1038 /$ ismej.2010.90

Meile, L., Le Blay, G., and Thierry, A. (2008). Safety assessment of dairy microorganisms: Propionibacterium and Bifidobacterium. Int. J. Food Microbiol. 126, 316-320. doi: 10.1016/j.ijfoodmicro.2007.08.019

Nagakura, Y., Naitoh, Y., Kamato, T., Yamano, M., and Miyata, K. (1996). Compounds possessing 5-HT3 receptor antagonistic activity inhibit intestinal propulsion in mice. Eur. J. Pharmacol. 311, 67-72. doi: 10.1016/ 0014-2999(96)00403-7
Possemiers, S., Grootaert, C., Vermeiren, J., Gross, G., Marzorati, M., Verstraete W., et al. (2009). The intestinal environment in health and disease-recent insights on the potential of intestinal bacteria to influence human health. Curr. Pharm. Design 15, 2051-2065. doi: 10.2174/138161209788489159

Qiu, Z., Li, N., Lu, X., Zheng, Z., Zhang, M., and Qiao, X. (2018a). Characterization of microbial community structure and metabolic potential using Illumina MiSeq platform during the black garlic processing. Food Res. Int. 106, 428-438. doi: 10.1016/j.foodres.2017.12.081

Qiu, Z., Lu, X., Li, N., Zhang, M., and Qiao, X. (2018b). Characterization of garlic endophytes isolated from the black garlic processing. MicrobiologyOpen 7, e00547. doi: 10.1002/mbo3.547

Quast, C., Pruesse, E., Yilmaz, P., Gerken, J., Schweer, T., Yarza, P., et al. (2012). The SILVA ribosomal RNA gene database project: improved data processing and web-based tools. Nucleic Acids Res. 41, D590-D596. doi: 10.1093/nar/ gks1219

Riley, M. A., and Wertz, J. E. (2002). Bacteriocin diversity: ecological and evolutionary perspectives. Biochimie 84, 357-364. doi: 10.1016/S0300-9084 (02)01421-9

Ríos-Covián, D., Ruas-Madiedo, P., Margolles, A., Gueimonde, M., De Los Reyesgavilán, C. G., and Salazar, N. (2016). Intestinal short chain fatty acids and their link with diet and human health. Front. Microbiol. 7:185:185. doi: 10.3389/ fmicb.2016.00185

Schulz, S., Röcken, C., Mawrin, C., Weise, W., Höllt, V., and Schulz, S. (2004). Immunocytochemical identification of VPAC1, VPAC2, and $\mathrm{PAC} 1$ receptors in normal and neoplastic human tissues with subtype-specific antibodies. Clin. Cancer Res. 10, 8235-8242. doi: 10.1158/1078-0432.CCR-04-0939

Umu, Ö.C., Bäuerl, C., Oostindjer, M., Pope, P. B., Hernández, P. E., PérezMartínez, G., et al. (2016). The potential of class II bacteriocins to modify gut microbiota to improve host health. PloS One 11, e0164036. doi: 10.1371/ journal.pone.0164036

Walsh, M. C., Gardiner, G. E., Hart, O. M., Lawlor, P. G., Daly, M., Lynch, B., et al. (2008). Predominance of a bacteriocin-producing Lactobacillus salivarius component of a five-strain probiotic in the porcine ileum and effects on host immune phenotype. FEMS Microbiol. Ecol. 64, 317-327. doi: 10.1111/j.15746941.2008.00454.x

Wang, L., Hu, L., Xu, Q., Yin, B., Fang, D., Wang, G., et al. (2017). Bifidobacterium adolescentis exerts strain-specific effects on constipation induced by loperamide in BALB/c mice. Int. J. Mol. Sci. 18:318. doi: 10.3390/ijms18020318

Yilmaz, P., Parfrey, L. W., Yarza, P., Gerken, J., Pruesse, E., Quast, C., et al. (2014). The SILVA and "all-species living tree project (LTP)" taxonomic frameworks. Nucleic Acids Res. 42, D643-D648. doi: 10.1093/nar/gkt1209

Zaharoni, H., Rimon, E., Vardi, H., Friger, M., Bolotin, A., and Shahar, D. R. (2011). Probiotics improve bowel movements in hospitalized elderly patientsThe proage study. J. Nutr. Health Aging 15, 215-220. doi: 10.1007/s12603-0100323-3

Zhao, Y., and Yu, Y. B. (2016). Intestinal microbiota and chronic constipation. Springerplus 5, 1130. doi: 10.1186/s40064-016-2821-1

Conflict of Interest: The authors declare that the research was conducted in the absence of any commercial or financial relationships that could be construed as a potential conflict of interest.

Copyright (C) 2021 Qiao, Qiu, Tian, Yu, Zhao, Zhang, Zhai and Chen. This is an openaccess article distributed under the terms of the Creative Commons Attribution License (CC BY). The use, distribution or reproduction in other forums is permitted, provided the original author(s) and the copyright owner(s) are credited and that the original publication in this journal is cited, in accordance with accepted academic practice. No use, distribution or reproduction is permitted which does not comply with these terms. 